\title{
Quaternionic Monge-Ampère operator for unbounded plurisubharmonic functions
}

\author{
Dongrui Wan ${ }^{1}$
}

Received: 17 March 2017 / Accepted: 19 July 2018 / Published online: 27 July 2018

(c) Fondazione Annali di Matematica Pura ed Applicata and Springer-Verlag GmbH Germany, part of Springer Nature 2018

\begin{abstract}
In this paper, we generalize the definition of the quaternionic Monge-Ampère operator to some unbounded plurisubharmonic functions, and we prove that the quaternionic Monge-Ampère operator is continuous on the monotonically decreasing sequences of plurisubharmonic functions. After introducing the generalized Lelong number of a positive current, Demailly's comparison theorems are showed. Moreover, we prove that the quaternionic Lelong-Jensen-type formula also holds for the unbounded plurisubharmonic function.
\end{abstract}

Keywords Monge-Ampère operator · Quaternionic plurisubharmonic function · Positive current

Mathematics Subject Classification $32 \mathrm{U} 15 \cdot 31 \mathrm{C} 10 \cdot 2 \mathrm{U} 40$

\section{Introduction}

Quaternionic analysis, which becomes an active and important direction of research in mathematics, has many applications in physics. Quaternionic manifolds play important roles in the supersymmetric theory in physics. The Baston operator in the quaternionic complexes appears in quantization of $N=2$ supergravity black holes. Recently, quaternionic version of Calabi-Yau conjucture, which has applications in superstring theory, has attracted many analysts to study on this problem. And they have obtained some results [4-6]. Relating to this problem, it is interesting to study the pluripotential theory, in particular to study the quaternionic Monge-Ampère operator, on quaternionic manifolds [3,5,6,14,23,25].

Let $\Omega$ be an open set in $n$-dimensional quaternionic space $\mathbb{H}^{n}$. The quaternionic MongeAmpère operator is defined as the Moore determinant of the quaternionic Hessian of $u$ :

Dongrui Wan

wandongrui@szu.edu.cn

1 College of Mathematics and Statistics, Shenzhen University, Room 415 Science and Technology

Building, Shenzhen 518060, People's Republic of China 


$$
\operatorname{det}(u)=\operatorname{det}\left[\frac{\partial^{2} u}{\partial \bar{q}_{j} \partial q_{k}}(q)\right]
$$

Alesker proved in [1] a quaternionic version of Chern-Levine-Nirenberg estimate and extended the definition of quaternionic Monge-Ampère operator to continuous quaternionic plurisubharmonic functions. Since it is inconvenient to use the Moore determinant, the studying of the quaternionic Monge-Ampère operator is much more difficult than that of the complex Monge-Ampère operator.

To define the quaternionic Monge-Ampère operator on general quaternionic manifolds, Alesker introduced in [3] an operator in terms of the Baston operator $\Delta$, which is the first operator of the quaternionic complex on quaternionic manifolds. The $n$th power of this operator is exactly the quaternionic Monge-Ampère operator when the manifold is flat. On the flat space $\mathbb{H}^{n}$, the Baston operator $\triangle$ is the first operator of 0-Cauchy-Fueter complex:

$$
0 \rightarrow C^{\infty}(\Omega, \mathbb{C}) \stackrel{\Delta}{\rightarrow} C^{\infty}\left(\Omega, \wedge^{2} \mathbb{C}^{2 n}\right) \stackrel{D}{\rightarrow} C^{\infty}\left(\Omega, \mathbb{C}^{2} \otimes \wedge^{3} \mathbb{C}^{2 n}\right) \rightarrow \cdots
$$

Wang [22] wrote down explicitly each operator of the $k$-Cauchy-Fueter complex in terms of real variables.

Motivated by this, the author and Wang introduced in [19] two first-order differential operators $d_{0}$ and $d_{1}$ acting on the quaternionic version of differential forms. And the second operator $D$ in (1.1) can be written as $D:=\left(\begin{array}{l}d_{0} \\ d_{1}\end{array}\right)$. The behavior of $d_{0}, d_{1}$ and $\Delta=d_{0} d_{1}$ is very similar to $\partial, \bar{\partial}$ and $\partial \bar{\partial}$ in several complex variables. The quaternionic Monge-Ampère operator can be defined as $(\Delta u)^{n}$ and has a simple explicit expression, which is much more convenient to use than the previous definition by using Moore determinant.

By introducing the quaternionic version of differential forms, the author and Wang defined in [19] the notions of closed positive forms and closed positive currents in the quaternionic case. We proved that $\Delta u$ is a closed positive 2-current for any quaternionic plurisubharmonic function $u$, and showed that when functions $u_{1}, \ldots, u_{k}$ are locally bounded, $\Delta u_{1} \wedge \cdots \wedge \Delta u_{k}$ is a well defined closed positive current and is continuous on decreasing sequences. Then we generalized Bedford-Taylor theory [7] in complex analysis to the quaternionic case, i.e., extend the definition of the quaternionic Monge-Ampère operator $(\Delta u)^{n}$ to locally bounded quaternionic plurisubharmonic function $u$ and prove that the operator is continuous on monotonically decreasing sequences of plurisubharmonic functions.

Quaternionic positive currents are discussed before in $[3,12]$ and closed currents are discussed in [6]. Our definition of closedness matches positivity well. Therefore we can extend several results of closed positive currents in several complex variables to the quaternionic case $[15,16,20]$. The results about the quaternionic Monge-Ampère operator and quaternionic closed positive currents proved by Wang and the author $[15,16,18-20]$ play key roles in this paper.

The purpose of this paper is to generalize the definition of the quaternionic Monge-Ampère operator to some unbounded plurisubharmonic functions and to prove that the quaternionic Monge-Ampère operator is continuous on the monotonically decreasing sequences of plurisubharmonic functions. In this paper, we use Demailly's methods for the complex Monge-Ampère operator (cf. [8-11]).

We define the unbounded locus $L(u)$ to be the set of points $q \in \Omega$ such that $u$ is unbounded in every neighborhood of $q$. 
Theorem 1.1 Let $u_{1}, \ldots, u_{k}$ be plurisubharmonic functions in $\Omega$ and $T$ be a closed positive $(2 n-2 p)$-current such that

$$
\mathcal{H}_{4 p-4 m+1}\left(L\left(u_{t_{1}}\right) \cap \cdots \cap L\left(u_{t_{m}}\right) \cap \operatorname{Supp} T\right)=0
$$

for all choices of indices $t_{1}<\cdots<t_{m}$ in $\{1, \ldots, k\}$ and $k \leq p$. Then the currents $u_{1} \triangle u_{2} \wedge$ $\cdots \wedge \Delta u_{k} \wedge T$ and $\Delta u_{1} \wedge \cdots \wedge \Delta u_{k} \wedge T$ are well defined and have locally finite mass in $\Omega$.

Theorem 1.2 Let $u^{1}, \ldots, u^{k}$ and T satisfy condition (1.2). Let $\left\{u_{j}^{1}\right\}_{j \in \mathbb{N}}, \ldots,\left\{u_{j}^{k}\right\}_{j \in \mathbb{N}}$ be decreasing sequences of plurisubharmonic functions in $\Omega$ such that $\lim _{j \rightarrow \infty} u_{j}^{i}=u^{i}$, pointwise in $\Omega, i=1, \ldots, k$. Then as $j \rightarrow \infty$,

$$
u_{j}^{1} \triangle u_{j}^{2} \wedge \cdots \wedge \Delta u_{j}^{k} \wedge T \longrightarrow \triangle u^{1} \Delta u^{2} \wedge \cdots \wedge \Delta u^{k} \wedge T \quad \text { weakly, }
$$

$$
\Delta u_{j}^{1} \wedge \cdots \wedge \Delta u_{j}^{k} \wedge T \longrightarrow \Delta u^{1} \wedge \cdots \wedge \Delta u^{k} \wedge T \quad \text { weakly. }
$$

Let $\Omega$ be a quaternionic strictly pseudoconvex domain, i.e., $\Omega$ has a strictly plurisubharmonic exhaustion function. For a closed positive $(2 n-2 p)$-current $T$, we define the generalized Lelong number $v(T, \varphi)$ of $T$ with respect to the weight $\varphi$ (the generalized Lelong number in the complex case was introduced by Demailly [11]). Define

$$
v(T, \varphi):=\int_{\{\varphi=-\infty\}} T \wedge(\Delta \varphi)^{p}=\lim _{r \rightarrow-\infty} v(T, \varphi, r),
$$

where

$$
v(T, \varphi, r)=\int_{B_{\varphi}(r)} T \wedge(\triangle \varphi)^{p},
$$

and

$$
B_{\varphi}(r):=\{q \in \Omega ; \varphi(q)<r\} .
$$

Here $\varphi: \Omega \rightarrow[-\infty,+\infty)$ is a continuous plurisubharmonic function (in general $\varphi$ may assume $-\infty$ as a value at some discrete points in $\Omega$ ), satisfying that, there exists a real number $R$ such that $B_{\varphi}(R) \Subset \Omega$. In particular, if one let $\varphi=-\|q-a\|^{-2}$, which is the fundamental solution of quaternionic Monge-Ampère operator, the generalized Lelong number $v(T, \varphi)$ is just the Lelong number $v_{a}(T)$ of $T$ at point $a$ that we introduced in [19].

As applications of the main theorems above, we obtain two Demailly's comparison theorems for the generalized Lelong number. Moreover, we prove that the Lelong-Jensen-type formula we established in [19, Theorem 5.1] also holds for the unbounded function. The Lelong-Jensen-type formula can be regarded as a higher-dimensional version of Poisson integral formula.

The paper is organized as follows. The results about quaternionic Monge-Ampère operator and quaternionic closed positive currents we need are collected in Sect. 2. Theorems 1.1 and 1.2 are proved in Sect. 3. In Sect. 4, we introduce the generalized Lelong number and its relationship with quaternionic boundary measure. Moreover, two Demailly's comparison theorems for the generalized Lelong number and the quaternionic version of Lelong-Jensentype formula are showed. 


\section{Preliminaries on quaternionic Monge-Ampère operator}

In this section, we are going to recall some basic definitions and conclusions of quaternionic Monge-Ampère operator and quaternionic closed positive currents following [19,20].

A real valued function $f: \mathbb{H}^{n} \rightarrow \mathbb{R}$ is called quaternionic plurisubharmonic ( $P S H$, for short) if it is upper semicontinuous and its restriction to any right quaternionic line is subharmonic (in the usual sense). Any quaternionic $P S H$ function is subharmonic (cf. [1,2,6] for more information about $P S H$ functions).

We use the well-known embedding of the quaternionic algebra $\mathbb{H}$ into $\operatorname{End}\left(\mathbb{C}^{2}\right)$ defined by

$$
x_{0}+x_{1} \mathbf{i}+x_{2} \mathbf{j}+x_{3} \mathbf{k} \mapsto\left(\begin{array}{cc}
x_{0}+\mathbf{i} x_{1} & -x_{2}-\mathbf{i} x_{3} \\
x_{2}-\mathbf{i} x_{3} & x_{0}-\mathbf{i} x_{1}
\end{array}\right) .
$$

Actually, we will use the conjugate embedding

$$
\begin{aligned}
\tau: \mathbb{H}^{n} \cong \mathbb{R}^{4 n} \hookrightarrow \mathbb{C}^{2 n \times 2}, \\
\left(q_{0}, \ldots, q_{n-1}\right) \mapsto \mathbf{z}=\left(z^{j \alpha}\right) \in \mathbb{C}^{2 n \times 2},
\end{aligned}
$$

$j=0,1, \ldots, 2 n-1, \alpha=0,1$, with

$$
\left(\begin{array}{cc}
z^{(2 l) 0} & z^{(2 l) 1} \\
z^{(2 l+1) 0} & z^{(2 l+1) 1}
\end{array}\right):=\left(\begin{array}{cc}
x_{4 l}-\mathbf{i} x_{4 l+1} & -x_{4 l+2}+\mathbf{i} x_{4 l+3} \\
x_{4 l+2}+\mathbf{i} x_{4 l+3} & x_{4 l}+\mathbf{i} x_{4 l+1}
\end{array}\right),
$$

for $q_{l}=x_{4 l}+\mathbf{i} x_{4 l+1}+\mathbf{j} x_{4 l+2}+\mathbf{k} x_{4 l+3}, l=0,1, \ldots, n-1$. Pulling back to the quaternionic space $\mathbb{H}^{n} \cong \mathbb{R}^{4 n}$ by the embedding above, we define on $\mathbb{R}^{4 n}$ first-order differential operators $\nabla_{j \alpha}$ as following:

$$
\left(\begin{array}{cc}
\nabla_{(2 l) 0} & \nabla_{(2 l) 1} \\
\nabla_{(2 l+1) 0} & \nabla_{(2 l+1) 1}
\end{array}\right):=\left(\begin{array}{cc}
\partial_{x_{4 l}}+\mathbf{i} \partial_{x_{4 l+1}} & -\partial_{x_{4 l+2}}-\mathbf{i} \partial_{x_{4 l+3}} \\
\partial_{x_{4 l+2}}-\mathbf{i} \partial_{x_{4 l+3}} & \partial_{x_{4 l}}-\mathbf{i} \partial_{x_{4 l+1}}
\end{array}\right) .
$$

$z^{k \beta}$ 's can be viewed as independent variables and $\nabla_{j \alpha}$ 's are derivatives with respect to these variables. The operators $\nabla_{j \alpha}$ 's play very important roles in the investigating of regular functions in several quaternionic variables [13,21-24].

Let $\wedge^{2 k} \mathbb{C}^{2 n}$ be the complex exterior algebra generated by $\mathbb{C}^{2 n}, 0 \leq k \leq n$. Fix a basis $\left\{\omega^{0}, \omega^{1}, \ldots, \omega^{2 n-1}\right\}$ of $\mathbb{C}^{2 n}$. Let $\Omega$ be a domain in $\mathbb{R}^{4 n}$. Define $d_{0}, d_{1}: C_{0}^{\infty}\left(\Omega, \wedge^{p} \mathbb{C}^{2 n}\right) \rightarrow$ $C_{0}^{\infty}\left(\Omega, \wedge^{p+1} \mathbb{C}^{2 n}\right)$ by

$$
\begin{aligned}
& d_{0} F=\sum_{k, I} \nabla_{k 0} f_{I} \omega^{k} \wedge \omega^{I}, \\
& d_{1} F=\sum_{k, I} \nabla_{k 1} f_{I} \omega^{k} \wedge \omega^{I}, \\
& \triangle F=d_{0} d_{1} F,
\end{aligned}
$$

for $F=\sum_{I} f_{I} \omega^{I} \in C_{0}^{\infty}\left(\Omega, \wedge^{p} \mathbb{C}^{2 n}\right)$, where the multi-index $I=\left(i_{1}, \ldots, i_{p}\right)$ and $\omega^{I}:=$ $\omega^{i_{1}} \wedge \cdots \wedge \omega^{i_{p}}$. Although $d_{0}, d_{1}$ are not exterior differential, their behavior is similar to the exterior differential.

Lemma 2.1 (Proposition 2.2 in [19])

(1) $d_{0} d_{1}=-d_{1} d_{0}$.

(2) $d_{0}^{2}=d_{1}^{2}=0$, thus $d_{0} \triangle=d_{1} \triangle=0$.

(3) For $F \in C_{0}^{\infty}\left(\Omega, \wedge^{p} \mathbb{C}^{2 n}\right), G \in C_{0}^{\infty}\left(\Omega, \wedge^{q} \mathbb{C}^{2 n}\right)$, we have 


$$
d_{\alpha}(F \wedge G)=d_{\alpha} F \wedge G+(-1)^{p} F \wedge d_{\alpha} G, \quad \alpha=0,1 .
$$

We say $F$ is closed if $d_{0} F=d_{1} F=0$. For $u_{1}, \ldots, u_{n} \in C^{2}, \Delta u_{1} \wedge \cdots \wedge \Delta u_{k}$ is closed, $k=1, \ldots, n$. Moreover, it follows easily from Lemma 2.1 that $\Delta u_{1} \wedge \cdots \wedge \Delta u_{n}$ satisfies the following remarkable identities:

$$
\begin{aligned}
& \triangle u_{1} \wedge \Delta u_{2} \wedge \cdots \wedge \Delta u_{n}=d_{0}\left(d_{1} u_{1} \wedge \Delta u_{2} \wedge \cdots \wedge \Delta u_{n}\right) \\
& \quad=-d_{1}\left(d_{0} u_{1} \wedge \Delta u_{2} \wedge \cdots \wedge \Delta u_{n}\right)=d_{0} d_{1}\left(u_{1} \Delta u_{2} \wedge \cdots \wedge \Delta u_{n}\right) \\
& =\triangle\left(u_{1} \Delta u_{2} \wedge \cdots \wedge \Delta u_{n}\right) .
\end{aligned}
$$

To write down the explicit expression, we define for a function $u \in C^{2}$,

$$
\begin{gathered}
\triangle_{i j} u:=\frac{1}{2}\left(\nabla_{i 0} \nabla_{j 1} u-\nabla_{i 1} \nabla_{j 0} u\right), \quad \Delta u=\sum_{i, j=0}^{2 n-1} \triangle_{i j} u \omega^{i} \wedge \omega^{j} . \\
\triangle u_{1} \wedge \cdots \wedge \triangle u_{n}=\sum_{i_{1}, j_{1}, \ldots} \triangle_{i_{1} j_{1}} u_{1} \ldots \triangle_{i_{n} j_{n}} u_{n} \omega^{i_{1}} \wedge \omega^{j_{1}} \wedge \cdots \wedge \omega^{i_{n}} \wedge \omega^{j_{n}} \\
=\sum_{i_{1}, j_{1}, \ldots} \delta_{01 \ldots(2 n-1)}^{i_{1} j_{1} \ldots i_{n} j_{n}} \triangle_{i_{1} j_{1}} u_{1} \ldots \triangle_{i_{n} j_{n}} u_{n} \Omega_{2 n},
\end{gathered}
$$

for $u_{1}, \ldots, u_{n} \in C^{2}$, where $\Omega_{2 n}$ is defined as

$$
\Omega_{2 n}:=\omega^{0} \wedge \omega^{1} \wedge \cdots \wedge \omega^{2 n-2} \wedge \omega^{2 n-1},
$$

and $\delta_{01 \ldots(2 n-1)}^{i_{1} j_{1} \ldots i_{n} j_{n}}:=$ the sign of the permutation from $\left(i_{1}, j_{1}, \ldots i_{n}, j_{n}\right)$ to $(0,1, \ldots, 2 n-1)$, if $\left\{i_{1}, j_{1}, \ldots, i_{n}, j_{n}\right\}=\{0,1, \ldots, 2 n-1\}$; otherwise, $\delta_{01 \ldots(2 n-1)}^{i_{1} j_{1} \ldots i_{n} j_{n}}=0$.

Note that $\Delta u_{1} \wedge \cdots \wedge \Delta u_{n}$ is symmetric with respect to the permutation of $u_{1}, \ldots, u_{n}$. In particular, when $u_{1}=\ldots=u_{n}=u, \Delta u_{1} \wedge \cdots \wedge \Delta u_{n}$ coincides with $(\triangle u)^{n}:=$ $\wedge^{n} \triangle u=\triangle_{n} u \Omega_{2 n}$. Denote by $\triangle_{n}\left(u_{1}, \ldots, u_{n}\right)$ the coefficient of the form $\Delta u_{1} \wedge \cdots \wedge \Delta u_{n}$, i.e., $\triangle u_{1} \wedge \cdots \wedge \Delta u_{n}=\triangle_{n}\left(u_{1}, \ldots, u_{n}\right) \Omega_{2 n}$. Then $\triangle_{n}\left(u_{1}, \ldots, u_{n}\right)$ coincides with the mixed Monge-Ampère operator det $\left(u_{1}, \ldots, u_{n}\right)$ while $\triangle_{n} u$ coincides with the quaternionic Monge-Ampère operator $\operatorname{det}(u)$. This result was proved by Alesker (Proposition 7.1 in [3]). And in Appendix A in [19] we gave an elementary and simpler proof of the identity:

$$
\triangle u_{1} \wedge \cdots \wedge \triangle u_{n}=n ! \operatorname{det}\left(u_{1}, \ldots, u_{n}\right) \Omega_{2 n}
$$

for $C^{2}$ function $u$.

We defined in [19] the notions of quaternionic positive forms and strongly positive forms. They are counterparts of forms in several complex variables. Please see [19] for detailed definitions. Denoted by $\mathcal{D}^{p}(\Omega)$ the set of all $C_{0}^{\infty}(\Omega)$ functions valued in $\wedge^{p} \mathbb{C}^{2 n}$. The elements of $\mathcal{D}^{p}(\Omega)$ are often called the test $p$-forms. An element of the dual space $\left(\mathcal{D}^{2 n-p}(\Omega)\right)^{\prime}$ is called a $p$-current. Denoted by $\mathcal{D}_{0}^{p}(\Omega)$ the set of all $C_{0}(\Omega)$ functions valued in $\wedge^{p} \mathbb{C}^{2 n}$. The elements of the dual space $\left(\mathcal{D}_{0}^{2 n-p}(\Omega)\right)^{\prime}$ are called $p$-currents of order zero. The $2 n$-currents are just the distributions on $\Omega$, whereas the $2 n$-currents of order zero are Radon measures on $\Omega$.

If $T$ is a $2 k$-current on $\Omega, \psi$ is a $2 l$-form on $\Omega$ with coefficients in $C^{\infty}(\Omega)$, and $k+l \leq n$, then the formula

$$
(T \wedge \psi)(\varphi)=T(\psi \wedge \varphi) \quad \text { for } \varphi \in \mathcal{D}^{2 n-2 k-2 l}(\Omega)
$$

defines a $(2 k+2 l)$-current. 
A $2 k$-current $T$ is said to be positive if we have $T(\eta) \geq 0$ for any strongly positive form $\eta \in \mathcal{D}^{2 n-2 k}(\Omega)$. Although a $2 n$-form is not an authentic differential form and we cannot integrate it, we can define

$$
\int_{\Omega} F:=\int_{\Omega} f \mathrm{~d} V
$$

if we write $F=f \Omega_{2 n} \in L^{1}\left(\Omega, \wedge^{2 n} \mathbb{C}^{2 n}\right)$, where $\mathrm{d} V$ is the Lebesgue measure and $\Omega_{2 n}$ is given by (2.4). In particular, if $F$ is positive $2 n$-form, then $\int_{\Omega} F \geq 0$. For a $2 n$-current $F=\mu \Omega_{2 n}$ with coefficient to be measure $\mu$, define

$$
\int_{\Omega} F:=\int_{\Omega} \mu \text {. }
$$

Any positive $2 k$-current $T$ on $\Omega$ has measure coefficients (i.e., is of order zero) (cf. Proposition 3.4 in [19] for details). For a positive $2 k$-current $T$ and a strongly positive test form $\varphi$, we can write $T \wedge \varphi=\mu \Omega_{2 n}$ for some Radon measure $\mu$. We have $T(\varphi)=\int_{\Omega} T \wedge \varphi$.

Lemma 2.2 (Stokes-type formula, Lemma 3.2 in [19]) Assume that $T=\sum_{i} T_{i} \omega^{\widehat{i}}$ is a smooth $(2 n-1)$-form in $\Omega$, where $\omega^{\hat{i}}=\omega^{0} \wedge \cdots \wedge \omega^{i-1} \wedge \omega^{i+1} \wedge \cdots \wedge \omega^{2 n-1}$. Then for smooth function $h$, we have

$$
\int_{\Omega} h d_{\alpha} T=-\int_{\Omega} d_{\alpha} h \wedge T+\sum_{i=0}^{2 n-1}(-1)^{i-1} \int_{\partial \Omega} h T_{i} n_{i \alpha} \mathrm{d} S,
$$

where $n_{i \alpha}, i=0,1, \ldots, 2 n-1, \alpha=0,1$, is defined by the matrix:

$$
\left(\begin{array}{cc}
n_{(2 l) 0} & n_{(2 l) 1} \\
n_{(2 l+1) 0} & n_{(2 l+1) 1}
\end{array}\right):=\left(\begin{array}{cc}
n_{4 l}+\boldsymbol{i} n_{4 l+1} & -n_{4 l+2}-\boldsymbol{i} n_{4 l+3} \\
n_{4 l+2}-\boldsymbol{i} n_{4 l+3} & n_{4 l}-\boldsymbol{i} n_{4 l+1}
\end{array}\right),
$$

$l=0,1, \ldots, n-1$. Here $\boldsymbol{n}=\left(n_{0}, n_{1}, \ldots, n_{4 n-1}\right)$ is the unit outer normal vector to $\partial \Omega$ and $\mathrm{d} S$ denotes the surface measure of $\partial \Omega$. In particular, if $h=0$ on $\partial \Omega$, we have

$$
\int_{\Omega} h d_{\alpha} T=-\int_{\Omega} d_{\alpha} h \wedge T, \quad \alpha=0,1
$$

Lemma 2.3 (Proposition 3.6 in [19]) Let $T$ be a $(2 n-1)$-current such that supp $T \subset \Omega$. If $d_{\alpha} T$ is a positive $2 n$-current, then $\int_{\Omega} d_{\alpha} T=0$.

Bedford-Taylor theory [7] in complex analysis can be generalized to the quaternionic case.

Lemma 2.4 (Proposition 3.9 in [19]) Let $u$ be a locally bounded PSH function and let $T$ be a closed positive $2 k$-current. Then $\triangle u \wedge T$ defined by

$$
\triangle u \wedge T:=\triangle(u T),
$$

i.e., $(\triangle u \wedge T)(\eta):=u T(\triangle \eta)$, for any test form $\eta$, is also a closed positive current. Inductively,

$$
\triangle u_{1} \wedge \cdots \wedge \Delta u_{p} \wedge T:=\triangle\left(u_{1} \Delta u_{2} \wedge \cdots \wedge \Delta u_{p} \wedge T\right)
$$

is a closed positive current, when $u_{1}, \ldots, u_{p} \in P S H \cap L_{l o c}^{\infty}(\Omega)$.

In particular, for $u_{1}, \ldots, u_{n} \in P S H \cap L_{l o c}^{\infty}(\Omega), \Delta u_{1} \wedge \cdots \wedge \Delta u_{n}=\mu \Omega_{2 n}$ for a well defined positive Radon measure $\mu$. 
For any strongly positive test $(2 n-2 p)$-form $\psi$ on $\Omega,(2.11)$ can be rewritten as

$$
\int_{\Omega} \Delta u_{1} \wedge \cdots \wedge \Delta u_{p} \wedge \psi=\int_{\Omega} u_{1} \Delta u_{2} \wedge \cdots \wedge \Delta u_{p} \wedge \Delta \psi
$$

where $u_{1}, \ldots, u_{p} \in P S H \cap L_{l o c}^{\infty}(\Omega)$. Since positive currents have measure coefficients, (2.12) also holds for strongly positive $\psi \in \mathcal{D}_{0}^{2 n-2 p}(\Omega)$ vanishing on the boundary.

Alesker gave a quaternionic version of Chern-Levine-Nirenberg estimate in Proposition 6.3 in [3]. And we gave an elementary and simpler proof in [19]. Set

$$
\beta_{n}:=\sum_{l=0}^{n-1} \omega^{2 l} \wedge \omega^{2 l+1}=\frac{1}{8} \triangle\left(\|q\|^{2}\right) .
$$

$\beta_{n}$ is a positive 2-form and $\beta_{n}^{n}=\wedge^{n} \beta_{n}=n ! \Omega_{2 n}$ is a strongly positive $2 n$-form. Let $T$ be a positive $(2 n-2 p)$-current and $K$ be an arbitrary compact subset. Define

$$
\|T\|_{K}:=\int_{K} T \wedge \beta_{n}^{p} .
$$

In particular, if $T$ is a positive $2 n$-current, $\|T\|_{K}$ coincides with $\int_{K} T$ defined by (2.7).

Lemma 2.5 (Chern-Levine-Nirenberg-type estimate, Proposition 3.10 in [19]) Let $\Omega$ be a domain in $\mathbb{H}^{n}$. Let $K, L$ be compact subsets of $\Omega$ such that $L$ is contained in the interior of $K$. Then there exists a constant $C$ depending only on $K, L, \Omega$ such that for any $u_{1}, \ldots u_{n} \in$ $P S H \cap C^{2}(\Omega)$, one has

$$
\left\|\Delta u_{1} \wedge \cdots \wedge \Delta u_{k}\right\|_{L} \leq C\left\|u_{1}\right\|_{L^{\infty}(K)} \cdots\left\|u_{k}\right\|_{L^{\infty}(K)} .
$$

The following convergence results are powerful tools in developing pluripotential theory for the quaternionic Monge-Ampère operator.

Lemma 2.6 (1) (Theorem 3.1 in [19])

Let $v^{1}, \ldots, v^{k} \in P S H \cap L_{\text {loc }}^{\infty}(\Omega)$. Let $\left\{v_{j}^{1}\right\}_{j \in \mathbb{N}}, \ldots,\left\{v_{j}^{k}\right\}_{j \in \mathbb{N}}$ be decreasing sequences of PSH functions in $\Omega$ such that $\lim _{j \rightarrow \infty} v_{j}^{t}=v^{t}$ pointwise in $\Omega$ for each $t$. Then the currents $\triangle v_{j}^{1} \wedge \cdots \wedge \Delta v_{j}^{k}$ converge weakly to $\Delta v^{1} \wedge \cdots \wedge \Delta v^{k}$ as $j \rightarrow \infty$.

(2) (Proposition 4.1 in [20])

Let $\left\{u_{j}\right\}_{j \in \mathbb{N}}$ be a sequence in $P S H \cap L_{\text {loc }}^{\infty}(\Omega)$ that increases to $u \in P S H \cap L_{\text {loc }}^{\infty}(\Omega)$ almost everywhere in $\Omega$ (with respect to Lebesgue measure). Then the currents $\left(\triangle u_{j}\right)^{n}$ converge weakly to $(\triangle u)^{n}$ as $j \rightarrow \infty$.

\section{Definition of wedge product $\Delta u_{1} \wedge \cdots \wedge \Delta u_{k} \wedge T$ for unbounded $P S H$ functions}

First we show that the Chern-Levine-Nirenberg-type estimate in Lemma 2.5 also holds for the closed current $\Delta u_{1} \wedge \cdots \wedge \Delta u_{k} \wedge T$ with closed positive current $T$.

Proposition 3.1 Let $\Omega$ be a domain in $\mathbb{H}^{n}$. Let $K, L$ be compact subsets of $\Omega$ such that $L$ is contained in the interior of $K$. Then there exists a constant $C$ depending only on $K, L$ such that for any $u_{1}, \ldots u_{k} \in P S H \cap C^{2}(\Omega)$ and closed positive $(2 n-2 p)$-current $T$ with $p \geq k$, one has

$$
\left\|\Delta u_{1} \wedge \cdots \wedge \Delta u_{k} \wedge T\right\|_{L} \leq C \prod_{i=1}^{k}\left\|u_{i}\right\|_{L^{\infty}(K)}\|T\|_{K},
$$


where $\|\cdot\|_{L}$ is defined by (2.14).

Proof By Lemma 2.4, $\Delta u_{1} \wedge \cdots \wedge \Delta u_{k} \wedge T$ is already a closed positive current. By the induction definition (2.11), it suffices to prove that

$$
\left\|\triangle u_{1} \wedge T\right\|_{L} \leq C\left\|u_{1}\right\|_{L^{\infty}(K)}\|T\|_{K} .
$$

Since $L$ is compact, there is a covering of $L$ by a family of balls $B_{j}^{\prime} \Subset B_{j} \subset K$. Let $\chi \geq 0$ be a smooth function equals to 1 on ${\overline{B_{j}}}^{\prime}$ with support in $B_{j}$. Then

$$
\begin{aligned}
\left\|\Delta u_{1} \wedge T\right\|_{L \cap{\overline{B_{j}}}^{\prime}} & =\int_{{\overline{B_{j}}}^{\prime}} \Delta u_{1} \wedge T \wedge \beta_{n}^{p-1} \leq \int_{B_{j}} \chi \Delta u_{1} \wedge T \wedge \beta_{n}^{p-1} \\
& =\left(T \wedge \beta_{n}^{p-1}\right)\left(\chi \triangle u_{1}\right)=\left(T \wedge \beta_{n}^{p-1}\right)\left(u_{1} \Delta \chi\right)=\int_{B_{j}} u_{1} \Delta \chi \wedge T \wedge \beta_{n}^{p-1} \\
& \leq \frac{1}{\varepsilon}\left\|u_{1}\right\|_{L^{\infty}(K)}\|\Delta \chi\| \int_{B_{j}} T \wedge \beta_{n}^{p},
\end{aligned}
$$

where the second identity follows from Proposition 3.8 in [19] and the last inequality follows from Lemma 3.1 below.

Lemma 3.1 (Lemma 3.3 in [19]) For each $\eta \in \wedge^{2 k} \mathbb{C}^{2 n *}, \beta_{n}^{k} \pm \varepsilon \eta$ is a positive $2 k$-form when $\varepsilon$ is sufficiently small.

Corollary 3.1 Let $u_{1}, \ldots, u_{s}, u_{1}^{(1)}, \ldots, u_{l}^{(1)}, u_{1}^{(2)}, \ldots, u_{l}^{(2)} \in P S H \cap L^{\infty}(\Omega), s+l \leq p$, and let $T$ be a closed positive $(2 n-2 p)$-current. Then the mixed product $\Theta=\Delta u_{1} \wedge \cdots \wedge$ $\triangle u_{s} \wedge \gamma\left(u_{1}^{(1)}, u_{1}^{(2)}\right) \wedge \cdots \wedge \gamma\left(u_{l}^{(1)}, u_{l}^{(2)}\right) \wedge T$ is also well defined, where

$$
\gamma\left(u_{t}^{(1)}, u_{t}^{(2)}\right):=d_{0} u_{t}^{(1)} \wedge d_{1} u_{t}^{(2)}+d_{0} u_{t}^{(2)} \wedge d_{1} u_{t}^{(1)}, \quad t=1, \ldots, l .
$$

And for $L \subset K^{o}$, we have

$$
\|\Theta\|_{L} \leq C\|T\|_{K} \prod_{m=1}^{s}\left\|u_{m}\right\|_{L^{\infty}(K)} \prod_{t=1}^{l}\left\|u_{t}^{(1)}\right\|_{L^{\infty}(K)}\left\|u_{t}^{(2)}\right\|_{L^{\infty}(K)} .
$$

Proof For each $t$, by Lemma 2.1(3) we have the polarization identity

$$
\begin{aligned}
& 2\left(d_{0} u_{t}^{(1)} \wedge d_{1} u_{t}^{(2)}+d_{0} u_{t}^{(2)} \wedge d_{1} u_{t}^{(1)}\right) \\
& \quad=d_{0} d_{1}\left(u_{t}^{(1)}+u_{t}^{(2)}\right)^{2}-d_{0} d_{1}\left(u_{t}^{(1)}\right)^{2}-d_{0} d_{1}\left(u_{t}^{(2)}\right)^{2}-2 u_{t}^{(1)} d_{0} d_{1} u_{t}^{(2)}-2 u_{t}^{(2)} d_{0} d_{1} u_{t}^{(1)} .
\end{aligned}
$$

And it follows that

$$
\begin{aligned}
2 \gamma\left(u_{t}^{(1)}, u_{t}^{(2)}\right) \wedge \Theta^{\prime}= & \Delta\left(u_{t}^{(1)}+u_{t}^{(2)}\right)^{2} \wedge \Theta^{\prime}-\Delta\left(u_{t}^{(1)}\right)^{2} \wedge \Theta^{\prime}-\Delta\left(u_{t}^{(2)}\right)^{2} \wedge \Theta^{\prime} \\
& -2 u_{t}^{(1)} \Delta u_{t}^{(2)} \wedge \Theta^{\prime}-2 u_{t}^{(2)} \Delta u_{t}^{(1)} \wedge \Theta^{\prime},
\end{aligned}
$$

where $\Theta^{\prime}=\Delta u_{1} \wedge \cdots \wedge \Delta u_{s} \wedge T$. Then (3.3) follows by applying estimates (3.1). Noting that all functions $u_{t}^{(\alpha)}, \alpha=0,1$, are bounded, we can let $u_{t}^{(\alpha)} \geq 0$ by adding a positive constant. So $\left(u_{t}^{(\alpha)}\right)^{2}$ is also plurisubharmonic. Therefore, $(3.1)$ can be applied to $\left(u_{t}^{(\alpha)}\right)^{2}$ and $\left(u_{t}^{(1)}+u_{t}^{(2)}\right)^{2}$. It is similar to handle with several $\gamma\left(u_{t}^{(1)}, u_{t}^{(2)}\right)^{\prime}$ s. 
When all functions are locally bounded, the mixed product $\gamma(u, u) \wedge \Delta w^{1} \wedge \cdots \wedge \Delta w^{k}$ is well defined as a positive $(2 k+2)$-current. Moreover, this positive current is also continuous on decreasing sequences (see Proposition 3.1 in [15] for more discussion about the mixed product).

Now we are going to show that $\triangle v_{1} \wedge \cdots \wedge \triangle v_{k} \wedge T$ is continuous on decreasing sequences when $v_{1}, \ldots, v_{k}$ are locally bounded $P S H$ functions and $T$ is a closed positive current. See this conclusion in the complex case in Demailly [11] and Bedford and Taylor [7].

Lemma 3.2 (Lemma 1.9 in [11]) Let $f_{j}$ be a decreasing sequence of upper semicontinuous functions converging to $f$ pointwise on some separable locally compact space $X$ and $\mu_{j}$ a sequence of positive measures converging weakly to $\mu$ on $X$. Then every weak limit $v$ of $f_{j} \mu_{j}$ satisfies $v \leq f \mu$.

Proposition 3.2 Let $v^{1}, \ldots, v^{k} \in P S H \cap L_{l o c}^{\infty}(\Omega)$ and let $T$ be a closed positive $(2 n-2 p)$ current with $p \geq k$. Let $\left\{v_{j}^{1}\right\}_{j \in \mathbb{N}}, \ldots,\left\{v_{j}^{k}\right\}_{j \in \mathbb{N}}$ be decreasing sequences of $P S H$ functions in $\Omega$ such that $\lim _{j \rightarrow \infty} v_{j}^{t}=v^{t}$, pointwise in $\Omega$, for $t=1, \ldots, k$. Then

$$
v_{j}^{1} \triangle v_{j}^{2} \wedge \cdots \wedge \Delta v_{j}^{k} \wedge T \longrightarrow v^{1} \Delta v^{2} \wedge \cdots \wedge \Delta v^{k} \wedge T \text { weakly }
$$

$$
\triangle v_{j}^{1} \wedge \cdots \wedge \Delta v_{j}^{k} \wedge T \longrightarrow \Delta v^{1} \wedge \cdots \wedge \Delta v^{k} \wedge T \quad \text { weakly }
$$

Proof Since the results are purely local, we can assume that $\Omega$ is an open ball $B=B(a, r)$ and the functions $v_{j}^{t}$ are smooth in $B$ and coincide outside a compact subset of $B$ as in the proof of Lemma 2.6(1) (Theorem 3.1 in [19]; see also Bedford and Taylor [7]).

Note that $(a) \Rightarrow(b)$ by the induction definition (2.11), and the case of $k=1$ for (a) is obvious. Suppose that $(a)$ has been proved for $k-1$. So we have

$$
S_{j}=\Delta v_{j}^{2} \wedge \cdots \wedge \Delta v_{j}^{k} \wedge T \longrightarrow S=\Delta v^{2} \wedge \cdots \wedge \Delta v^{k} \wedge T \text { weakly. }
$$

To prove ( $a$ ) for $k$, it suffices to show that the weak limit $\Theta$ of $v_{j}^{1} S_{j}$ is equal to $v^{1} S$. Let $\eta$ be a strongly positive test $(2 p-2 k+2)$-form. Then $S_{j} \wedge \eta$ converges weakly to $S \wedge \eta$. Denote $S_{j} \wedge \eta=\mu_{j} \Omega_{2 n}$ and $S \wedge \eta=\mu \Omega_{2 n}$ with $\mu_{j}, \mu$ positive measures. Apply Lemma 3.2 to $v_{j}^{1}, v^{1}, \mu_{j}, \mu$ to get $\Theta \wedge \eta \leq v^{1} S \wedge \eta$. Thus the weak limit $\Theta \leq v^{1} S$. To prove the equality, it suffices to show that

$$
\int_{B} v^{1} \Delta v^{2} \wedge \cdots \wedge \Delta v^{k} \wedge T \wedge \beta_{n} \leq \liminf _{j \rightarrow \infty} \int_{B} v_{j}^{1} \Delta v_{j}^{2} \wedge \cdots \wedge \Delta v_{j}^{k} \wedge T \wedge \beta_{n}
$$

for $\beta_{n}=\frac{1}{8} \Delta \rho, \rho(q)=\|q-a\|^{2}-r^{2}$.

Since the functions $v_{j}^{t}$ are bounded on the ball $B$, we can also assume that $-M<v_{j}^{t}<-1$ after adding a constant. Then for large $A$ the function $\max \left(v_{j}^{t}, A \rho\right)$ will coincide with $A \rho$ near the boundary and remain the same near the center. Therefore we can assume that all $v_{j}^{t}$ are smooth after regularization and vanish on the boundary. Now since $v^{1} \leq v_{j}^{1}$, by Lemma 2.1 
we have

$$
\begin{aligned}
\int_{B} v^{1} \Delta v^{2} \wedge \cdots \wedge \Delta v^{k} \wedge T \wedge \beta_{n} & \leq \int_{B} v_{j}^{1} \Delta v^{2} \wedge \cdots \wedge \Delta v^{k} \wedge T \wedge \beta_{n} \\
& =\int_{B} v_{j}^{1} d_{0} d_{1} v^{2} \wedge \Delta v^{3} \wedge \cdots \wedge \Delta v^{k} \wedge T \wedge \beta_{n} \\
& =-\int_{B} d_{0} v_{j}^{1} \wedge d_{1} v^{2} \wedge \Delta v^{3} \wedge \cdots \wedge \Delta v^{k} \wedge T \wedge \beta_{n} \\
& =\int_{B} d_{1} v^{2} \wedge d_{0} v_{j}^{1} \wedge \Delta v^{3} \wedge \cdots \wedge \Delta v^{k} \wedge T \wedge \beta_{n} \\
& =-\int_{B} v^{2} d_{1} d_{0} v_{j}^{1} \wedge \Delta v^{3} \wedge \cdots \wedge \Delta v^{k} \wedge T \wedge \beta_{n} \\
& =\int_{B} v^{2} \Delta v_{j}^{1} \wedge \Delta v^{3} \wedge \cdots \wedge \Delta v^{k} \wedge T \wedge \beta_{n} \\
& \leq \int_{B} v_{j}^{2} \Delta v_{j}^{1} \wedge \Delta v^{3} \wedge \cdots \wedge \Delta v^{k} \wedge T \wedge \beta_{n} \\
& =\ldots=\int_{B} v_{j}^{1} \Delta v_{j}^{2} \wedge \Delta v^{3} \wedge \cdots \wedge \Delta v^{k} \wedge T \wedge \beta_{n} .
\end{aligned}
$$

Here we use Stokes-type formula (2.9) for $h=v_{j}^{1}$ in the second identity and for $h=v^{2}$ in the fourth identity. Repeating this argument to obtain

$$
\int_{B} v^{1} \Delta v^{2} \wedge \cdots \wedge \Delta v^{k} \wedge T \wedge \beta_{n} \leq \int_{B} v_{j}^{1} \Delta v_{j}^{2} \wedge \cdots \wedge \Delta v_{j}^{k} \wedge T \wedge \beta_{n} .
$$

Finally it suffices to let $j \rightarrow \infty$.

The proof of Theorem 1.1 is also an induction on $k$, similar to the inductive process in the proof of Lemma 2.4. All that we need is the following Proposition 3.3, which is an improved version of Chern-Levine-Nirenberg-type estimate. To prove this proposition, we need the following lemma (cf. Lemma 2.7 in [11] for the proof in the complex case).

Lemma 3.3 Let $F \subset \mathbb{H}^{n}$ be a closed set such that $\mathcal{H}_{4 s+1}(F)=0$ for some integer $0 \leq$ $s<n$. Then for almost all choices of unitary coordinates $\left(q_{1}, \ldots, q_{n}\right)=\left(q^{\prime}, q^{\prime \prime}\right)$ with $q^{\prime}=$ $\left(q_{1}, \ldots, q_{s}\right), q^{\prime \prime}=\left(q_{s+1}, \ldots, q_{n}\right)$ and almost all radii of balls $B^{\prime \prime}=B\left(0, r^{\prime \prime}\right) \subset \mathbb{H}^{n-s}$, the set $\{0\} \times \partial B^{\prime \prime}$ does not intersect $F$.

Proof The unitary group $U_{\mathbb{H}}(n)$ has real dimension $2 n^{2}+n$ by calculating the dimension of its Lie algebra \{quaternionic $(n \times n)-$ matrix $\left.A ; A+A^{*}=0\right\}$. There is a proper submersion

$$
\Phi: U_{\mathbb{H}}(n) \times\left(\mathbb{H}^{n-s} \backslash\{0\}\right) \rightarrow \mathbb{H}^{n} \backslash\{0\}, \quad\left(g, q^{\prime \prime}\right) \mapsto g\left(0, q^{\prime \prime}\right),
$$

whose fibers have real dimension $N=2 n^{2}+n-4 s$. It follows that the inverse image $\Phi^{-1}(F)$ has zero Hausdorff measure $\mathcal{H}_{N+4 s+1}=\mathcal{H}_{2 n^{2}+n+1}$. The set of pairs $\left(g, r^{\prime \prime}\right) \in U_{\mathbb{H}}(n) \times \mathbb{R}_{+}^{*}$ such that $g\left(\{0\} \times \partial B^{\prime \prime}\right)$ intersects $F$ is precisely the image of $\Phi^{-1}(F)$ in $U_{\mathbb{H}}(n) \times \mathbb{R}_{+}^{*}$ by the Lipschitz map $\left(g, q^{\prime \prime}\right) \mapsto\left(g,\left|q^{\prime \prime}\right|\right)$. Hence this set has zero $\mathcal{H}_{2 n^{2}+n+1}$-measure.

We define the unbounded locus $L(u)$ to be the set of points $q \in \Omega$ such that $u$ is unbounded in every neighborhood of $q$. 
Proposition 3.3 Let $u_{1}, \ldots, u_{k}$ be PSH functions in $\Omega$ and let $T$ be a closed positive ( $2 n-$ $2 p$ )-current, $k \leq p$. Let $U_{1}, \ldots, U_{k} \subset \Omega$ be closed sets such that

$$
\mathcal{H}_{4 p-4 m+1}\left(U_{t_{1}} \cap \cdots \cap U_{t_{m}} \cap \operatorname{Supp} T\right)=0
$$

for all choices of indices $t_{1}, \ldots, t_{m}$ in $\{1, \ldots, k\}$. Then for all compact sets $K, L$ of $\Omega$ with $L \subset K^{o}$, there exist neighborhoods $V_{t}$ of $K \cap U_{t}, t=1, \ldots, k$, and a constant $C=C\left(K, L, U_{1}, \ldots, U_{k}\right)$ such that the conditions $u_{t} \leq 0$ on $K$ and $L\left(u_{t}\right) \subset U_{t}$ imply

$$
\begin{gathered}
\left\|u_{1} \Delta u_{2} \wedge \cdots \wedge \Delta u_{k} \wedge T\right\|_{L} \leq C\left\|u_{1}\right\|_{L^{\infty}\left(K \backslash V_{1}\right)} \cdots\left\|u_{k}\right\|_{L^{\infty}\left(K \backslash V_{k}\right)}\|T\|_{K} \\
\left\|\Delta u_{1} \wedge \cdots \wedge \Delta u_{k} \wedge T\right\|_{L} \leq C\left\|u_{1}\right\|_{L^{\infty}\left(K \backslash V_{1}\right)} \cdots\left\|u_{k}\right\|_{L^{\infty}\left(K \backslash V_{k}\right)}\|T\|_{K} .
\end{gathered}
$$

Proof It is sufficient to show that every point $x_{0} \in K^{o}$ has a neighborhood $L$ such that $(a),(b)$ hold. After a mollification process $u_{t}=\lim _{\varepsilon \rightarrow 0} u_{t} * \chi_{\varepsilon}$ for $t=k, k-1, \ldots, 1$ in this order, we may assume that $u_{1}, \ldots, u_{k}$ are smooth. We proceed by induction on $k$ in two steps:

Step $1 \quad\left(b_{k-1}\right) \Rightarrow b_{k}$,

Step $2\left(a_{k-1}\right)$ and $\left(b_{k}\right) \Rightarrow\left(a_{k}\right)$.

First $\left(b_{0}\right)$ is the trivial statement $\|T\|_{L} \leq\|T\|_{K}$. Let $x_{0}=0 \in K^{o}$ and $L$ be a neighborhood of 0 . To prove Step 1, we need to show that

$$
\int_{L} \Delta u_{1} \wedge \cdots \wedge \Delta u_{k} \wedge T \wedge \beta_{n}^{s} \leq C\left\|u_{1}\right\|_{L^{\infty}\left(K \backslash V_{1}\right)} \cdots\left\|u_{k}\right\|_{L^{\infty}\left(K \backslash V_{k}\right)}\|T\|_{K}
$$

with $s=p-k$. By Corollary 2.2 in [19], if we change the unitary basis in $\mathbb{H}^{n}, \triangle u_{t}$ is invariant. So we only need to show the estimate (3.8) for $\triangle u_{1} \wedge \cdots \wedge \Delta u_{k} \wedge T \wedge \omega^{0} \wedge \omega^{1} \wedge \cdots \wedge \omega^{2 s-1}$.

Suppose first $0 \in U_{1} \cap \cdots \cap U_{k}$ and set $F=U_{1} \cap \cdots U_{k} \cap \operatorname{Supp} T$. Since $\mathcal{H}_{4 p-4 k+1}(F)=0$, by Lemma 3.3 above, there are coordinates $q^{\prime}=\left(q_{1}, \ldots, q_{s}\right), q^{\prime \prime}=\left(q_{s+1}, \ldots, q_{n}\right)$ and a ball $\bar{B}^{\prime \prime}$ such that $F \cap\left(\{0\} \times \partial B^{\prime \prime}\right)=\emptyset$ and $\{0\} \times \bar{B}^{\prime \prime} \subset K^{o}$. Since $K$ is compact, we can find neighborhoods $W_{t}$ of $K \cap U_{t}$ and a ball $B^{\prime}=B\left(0, r^{\prime}\right) \subset \mathbb{H}^{s}$ such that $\bar{B}^{\prime} \times \bar{B}^{\prime \prime} \subset K^{o}$ and

$$
\bar{W}_{1} \cap \cdots \cap \bar{W}_{k} \cap \operatorname{Supp} T \cap\left(\bar{B}^{\prime} \times\left(\bar{B}^{\prime \prime} \backslash(1-\delta) \bar{B}^{\prime \prime}\right)\right)=\emptyset
$$

for $\delta>0$ small.

When $0 \notin U_{t}$ for some $t$, we set instead $W_{t}$ to be a small neighborhood of 0 such that $\bar{W}_{t} \subset\left(\bar{B}^{\prime} \times(1-\delta) \bar{B}^{\prime \prime}\right) \backslash U_{t}$, then (3.9) is satisfied automatically. Let $\chi_{t} \geq 0$ be a function with compact support in $W_{t}$, equal to 1 near $K \cap U_{t}$ if $0 \in U_{t}$ (resp. equal to 1 near 0 if $\left.0 \notin U_{t}\right)$. Let $\chi\left(q^{\prime}\right) \geq 0$ be a function equal to 1 on $\frac{1}{2} B^{\prime}$ with compact support in $B^{\prime}$.

Note that $\widetilde{\alpha}:=\chi\left(q^{\prime}\right)\left(\triangle\left(\left\|q^{\prime}\right\|^{2}\right)\right)^{s}=\chi\left(q^{\prime}\right) 8^{s} s ! \omega^{0} \wedge \omega^{1} \wedge \cdots \wedge \omega^{2 s-1}$ is closed, where $q^{\prime}=\left(q_{1}, \ldots, q_{s}\right)$. This is because, 


$$
d_{\alpha} \tilde{\alpha}=8^{s} s ! \sum_{k=0}^{2 n-1} \nabla_{k \alpha}\left(\chi\left(q^{\prime}\right)\right) \omega^{k} \wedge \omega^{0} \wedge \omega^{1} \wedge \cdots \wedge \omega^{2 s-1}=0,
$$

for $\alpha=0,1$, by considering the type and $\nabla_{k \alpha}\left(\chi\left(q^{\prime}\right)\right) \equiv 0$ for $k=2 s, \ldots, 2 n-1$. Since $T$ and $\tilde{\alpha}$ are closed, we find that

$$
\triangle\left(\chi_{1} u_{1}\right) \wedge \cdots \wedge \triangle\left(\chi_{k} u_{k}\right) \wedge T \wedge \tilde{\alpha}=d_{0}\left[d_{1}\left(\chi_{1} u_{1}\right) \wedge \cdots \wedge \Delta\left(\chi_{k} u_{k}\right) \wedge T \wedge \tilde{\alpha}\right] .
$$

Then it follows from Lemma 2.3 and (3.9) that

$$
\begin{aligned}
& \int_{B^{\prime} \times B^{\prime \prime}} \Delta\left(\chi_{1} u_{1}\right) \wedge \cdots \wedge \Delta\left(\chi_{k} u_{k}\right) \wedge T \wedge \widetilde{\alpha} \\
& \quad=\int_{B^{\prime} \times B^{\prime \prime}} d_{0}\left[d_{1}\left(\chi_{1} u_{1}\right) \wedge \cdots \wedge \Delta\left(\chi_{k} u_{k}\right) \wedge T \wedge \tilde{\alpha}\right]=0 .
\end{aligned}
$$

The last identity holds because $d_{1}\left(\chi_{1} u_{1}\right) \wedge \cdots \wedge \triangle\left(\chi_{k} u_{k}\right) \wedge T \wedge \widetilde{\alpha}$ vanishes near the boundary of $B^{\prime} \times B^{\prime \prime}$ by (3.9).

If we expand all factors $\Delta\left(\chi_{t} u_{t}\right)$, we find a term

$$
\chi_{1} \ldots \chi_{k} \Delta u_{1} \wedge \cdots \wedge \Delta u_{k} \wedge T=\Delta u_{1} \wedge \cdots \wedge \Delta u_{k} \wedge T \geq 0
$$

on a neighborhood of 0 where $\chi_{1}=\ldots=\chi_{k}=1$. Other terms involve $\gamma\left(\chi_{t}, u_{t}\right)+u_{t} \Delta \chi_{t}$ for at least one index $t$, where $\gamma\left(\chi_{t}, u_{t}\right)=d_{0} \chi_{t} \wedge d_{1} u_{t}+d_{0} u_{t} \wedge d_{1} \chi_{t}$ is the mixed term. However all derivatives of $\chi_{t}$ vanish on some neighborhood $V_{t}^{\prime}$ of $K \cap U_{t}$. And note that $u_{t}$ is bounded on $\left(\bar{B}^{\prime} \times \bar{B}^{\prime \prime}\right) \backslash V_{t}^{\prime}$ by the fact that $L\left(u_{t}\right) \subset U_{t}$.

Let $K^{\prime}$ be a compact neighborhood of $\bar{B}^{\prime} \times \bar{B}^{\prime \prime}$ with $K^{\prime} \subset K^{o}$, and let $V_{t}$ be a neighborhood of $K \cap U_{t}$ with $\bar{V}_{t} \subset V_{t}^{\prime}$. To control these forms, for $L^{\prime}:=\left(\bar{B}^{\prime} \times \bar{B}^{\prime \prime}\right) \backslash V_{t}^{\prime} \subset\left(K^{\prime} \backslash V_{t}\right)^{o}$, we apply the usual Chern-Levine-Nirenberg-type estimate in Corollary 3.1 to the product of the positive current $\Theta=\Delta u_{1} \wedge \cdots \wedge \widehat{\triangle u_{t}} \wedge \cdots \wedge \Delta u_{k} \wedge T$ with the mixed term $\gamma\left(\chi_{t}, u_{t}\right)$ to get

$$
\left\|\gamma\left(\chi_{t}, u_{t}\right) \wedge \Theta\right\|_{\bar{B}^{\prime} \times \bar{B}^{\prime \prime}}=\left\|\gamma\left(\chi_{t}, u_{t}\right) \wedge \Theta\right\|_{L^{\prime}} \leq C_{1}\left\|u_{t}\right\|_{L^{\infty}\left(K^{\prime} \backslash V_{t}\right)}\|\Theta\|_{K^{\prime} \backslash V_{t}} .
$$

Then apply the induction hypothesis $\left(b_{k-1}\right)$ to the current $\Theta$ to get

$$
\|\Theta\|_{K^{\prime} \backslash V_{t}} \leq\|\Theta\|_{K^{\prime}} \leq C_{2}\left\|u_{1}\right\|_{K \backslash V_{1}} \ldots \widehat{\left\|u_{t}\right\|} \ldots\left\|u_{k}\right\|_{K \backslash V_{k}}\|T\|_{K} .
$$

Thus the mixed terms are uniformly bounded. For the same reason, the terms involving $u_{t} \Delta \chi_{t}$ are also uniformly bounded. For the terms involving several copies of $\gamma\left(\chi_{t}, u_{t}\right)$ and $u_{t} \Delta \chi_{t}$, one can get the desired bounds by repeating the above process. Finally, it follows from (3.12) and (3.13) that $\Delta u_{1} \wedge \cdots \wedge \Delta u_{k} \wedge T \wedge \omega^{0} \wedge \omega^{1} \wedge \cdots \wedge \omega^{2 s-2} \wedge \omega^{2 s-1}$ satisfies estimate $\left(b_{k}\right)$ on a neighborhood of 0 . Thus Step 1 is finished.

Now we proceed by induction to prove Step 2. Note that $d_{\alpha}\left(\chi\left(q^{\prime}\right)\left(\triangle\left(\left\|q^{\prime}\right\|^{2}\right)\right)^{S}\right)=0$ in (3.10). By applying Lemma 2.3 twice, we get

$$
\begin{aligned}
& \int_{\bar{B}^{\prime} \times \bar{B}^{\prime \prime}} \chi_{1} u_{1} \Delta\left(\chi_{2} u_{2}\right) \wedge \cdots \wedge \triangle\left(\chi_{k} u_{k}\right) \wedge T \wedge \chi\left(q^{\prime}\right)\left(\triangle\left(\left\|q^{\prime}\right\|^{2}\right)\right)^{s} \wedge \Delta\left(\left|q_{s+1}\right|^{2}\right) \\
& =\int_{\bar{B}^{\prime} \times \bar{B}^{\prime \prime}}\left|q_{s+1}\right|^{2} \triangle\left(\chi_{1} u_{1}\right) \wedge \triangle\left(\chi_{2} u_{2}\right) \wedge \cdots \wedge \triangle\left(\chi_{k} u_{k}\right) \wedge T \wedge \chi\left(q^{\prime}\right)\left(\triangle\left(\left\|q^{\prime}\right\|^{2}\right)\right)^{s} .
\end{aligned}
$$

By Corollary 3.1 and $\left(b_{k}\right)$, we already know that all terms in the right hand side integral admit the desired bound. And this also means $\left(b_{1}\right) \Rightarrow\left(a_{1}\right)$.

Except for $\chi_{1} \ldots \chi_{k} u_{1} \Delta u_{2} \wedge \cdots \wedge \Delta u_{k} \wedge T$, all terms in the left hand side of (3.14) involve derivatives of $\chi_{t}$ for at least one index $t, t \geq 2$. Note that all terms in the right hand 
side integral admit the desired bound. Once one can show that the terms in the left hand side involving derivatives of $\chi_{t}$ for at least one index $t, t \geq 2$, admit the desired bound, then it follows the desired bound for the integral of $\chi_{1} \ldots \chi_{k} u_{1} \Delta u_{2} \wedge \cdots \wedge \Delta u_{k} \wedge T$, which equals $u_{1} \Delta u_{2} \wedge \cdots \wedge \Delta u_{k} \wedge T$ on a neighborhood of 0 . Since the support of derivatives of $\chi_{t}, t \geq 2$, is disjoint from $U_{t}$, the integral involving these derivatives is actually on some neighborhood of 0 which is disjoint from $U_{t}$. Therefore we only have to obtain a bound for $\left\|u_{1} \Delta u_{2} \wedge \cdots \wedge \Delta u_{k} \wedge T \wedge \alpha^{\prime}\right\|_{L}$ when $L:=\bar{B}(0, r)$ is disjoint from $U_{t}$ for some $t \geq 2$, say $L \cap U_{2}=\emptyset$, and $\alpha^{\prime}$ is a constant positive $(2 p-2 k+2)$-form.

Then for some $\varepsilon>0$, and some neighborhood $V_{2}$ of $K \cap U_{2}$, we have $\bar{B}\left(x_{0}, r+\varepsilon\right) \subset$ $K^{o} \backslash \bar{V}_{2}$. Replace $u_{2}$ by a $P S H$ function $\widetilde{u}_{2}$, which equals to $u_{2}$ in $L$ and to $\frac{M}{\varepsilon r}\left(\|q\|^{2}-r^{2}\right)-M$ in $\bar{B}(0, r+\varepsilon) \backslash B\left(0, r+\frac{\varepsilon}{2}\right)$, with $M=\left\|u_{2}\right\|_{L^{\infty}\left(K \backslash V_{2}\right)}$. Let $\chi \geq 0$ be a smooth function equal to 1 in $B(0, r)$ with support in $B\left(0, r+\frac{\varepsilon}{2}\right)$. Then $\chi \widetilde{u}_{2}$ equal to $u_{2}$ in $L$ and equal to 0 out of $B\left(0, r+\frac{\varepsilon}{2}\right)$, and $\left\|\chi \tilde{u}_{2}\right\|_{B\left(0, r+\frac{\varepsilon}{2}\right)} \leq O(1)\left\|u_{2}\right\|_{L^{\infty}\left(K \backslash V_{2}\right)}$. By Lemma 2.3, we get

$$
\begin{aligned}
& \int_{B(0, r+\varepsilon)} u_{1} \Delta\left(\chi \tilde{u}_{2}\right) \wedge \Delta u_{3} \wedge \cdots \wedge \Delta u_{k} \wedge T \wedge \alpha^{\prime} \\
& \quad=\int_{B(0, r+\varepsilon / 2)} \chi \tilde{u}_{2} \Delta\left(u_{1}\right) \wedge \Delta u_{3} \wedge \cdots \wedge \Delta u_{k} \wedge T \wedge \alpha^{\prime} \\
& \quad \leq O(1)\left\|u_{1}\right\|_{L^{\infty}\left(K \backslash V_{1}\right)} \cdots\left\|u_{k}\right\|_{L^{\infty}\left(K \backslash V_{k}\right)}\|T\|_{K},
\end{aligned}
$$

where the last estimate follows from $\left(b_{k-1}\right)$ applied to $\Delta u_{1} \wedge \Delta u_{3} \wedge \cdots \wedge \Delta u_{k} \wedge T$. Note that

$$
\triangle\left(\chi \tilde{u}_{2}\right)=\chi \triangle \tilde{u}_{2}+(\text { smooth terms involving derivatives of } \chi)
$$

coincides with $\Delta u_{2}$ in $L$, and $\left(a_{k-1}\right)$ implies the required estimate for the other terms in the left hand side of (3.15). Then we get finally the desired bound.

Proof of Theorem 1.2 As in the proof of Proposition 3.3, we take $x_{0}=0$. We proceed by induction on $k$ and also on the number $m$ of functions $u^{t}, t=1, \ldots, k$, which are unbounded near the given point $x_{0}$. By Lemma 3.2 and the arguments already used in the proof of Proposition 3.2 , it suffices to prove that

$$
\begin{aligned}
& \int_{B^{\prime} \times B^{\prime \prime}} \chi_{1} \ldots \chi_{k} u^{1} \Delta u^{2} \wedge \cdots \wedge \Delta u^{k} \wedge T \wedge \alpha \\
& \quad \leq \liminf _{j \rightarrow \infty} \int_{B^{\prime} \times B^{\prime \prime}} \chi_{1} \ldots \chi_{k} u_{j}^{1} \Delta u_{j}^{2} \wedge \cdots \wedge \Delta u_{j}^{k} \wedge T \wedge \alpha,
\end{aligned}
$$

where $\alpha=\chi\left(q^{\prime}\right)\left(\triangle\left(\left\|q^{\prime}\right\|^{2}\right)\right)^{s}$, and $B^{\prime}, B^{\prime \prime}$ and the functions $\chi_{t}, t=1, \ldots, k$, are chosen as in the proof of Step 1 in Proposition 3.3. There product $\chi_{1} \ldots \chi_{k}$ has compact support in $B^{\prime} \times B^{\prime \prime}$ and $\chi_{1}=\ldots=\chi_{k}=1$ in a neighborhood of $x_{0}$. If $u^{t}$ is bounded near $x_{0}$, we take $W_{t}^{\prime \prime} \Subset W_{t}^{\prime} \Subset W_{t}$ to be small balls of center $x_{0}$ on which $u^{t}$ is bounded and we modify the sequence $u_{j}^{t}$ on the ring $W_{t} \backslash W_{t}^{\prime \prime}$ so as to make it constant and equal to a smooth function $A\left\|q-x_{0}\right\|^{2}+B$ on the smaller ring $W_{t} \backslash W_{t}^{\prime}$. In this case, we take $\chi_{t}$ equal to 1 near $\bar{W}_{t}^{\prime}$ and Supp $\chi_{t} \subset W_{t}$. 
For every $l=1, \ldots, k$, as the process in (3.5), we replace $\chi_{1} u_{j}^{1} \triangle\left(\chi_{l} u^{l}\right)$ by $\chi_{l} u^{l} \triangle\left(\chi_{1} u_{j}^{1}\right)$ and use the inequality $u^{l} \leq u_{j}^{l}$ to get

$$
\begin{aligned}
& \liminf _{j \rightarrow \infty} \int_{B^{\prime} \times B^{\prime \prime}} \chi_{1} u_{j}^{1} \Delta\left(\chi_{2} u_{j}^{2}\right) \wedge \cdots \wedge \Delta\left(\chi_{l-1} u_{j}^{l-1}\right) \wedge \\
& \quad \triangle\left(\chi_{l} u^{l}\right) \wedge \Delta\left(\chi_{l+1} u^{l+1}\right) \wedge \cdots \wedge \Delta\left(\chi_{k} u^{k}\right) \wedge T \wedge \alpha \\
& \leq \liminf _{j \rightarrow \infty} \int_{B^{\prime} \times B^{\prime \prime}} \chi_{1} u_{j}^{1} \triangle\left(\chi_{2} u_{j}^{2}\right) \wedge \cdots \wedge \Delta\left(\chi_{l-1} u_{j}^{l-1}\right) \wedge \\
& \quad \triangle\left(\chi_{l} u_{j}^{l}\right) \wedge \triangle\left(\chi_{l+1} u^{l+1}\right) \wedge \cdots \wedge \triangle\left(\chi_{k} u^{k}\right) \wedge T \wedge \alpha .
\end{aligned}
$$

If we expand all factors $\Delta\left(\chi_{t} u^{t}\right)$ in (3.17), we find an inequality involving only the term $\chi_{t} \Delta u^{t}$, that is,

$$
\begin{aligned}
& \liminf _{j \rightarrow \infty} \int_{B^{\prime} \times B^{\prime \prime}} \chi_{1} \ldots \chi_{k} u_{j}^{1} \Delta u_{j}^{2} \wedge \cdots \wedge \Delta u_{j}^{l-1} \wedge \Delta u^{l} \wedge \Delta u^{l+1} \wedge \cdots \wedge \Delta u^{k} \wedge T \wedge \alpha \\
& \quad \leq \liminf _{j \rightarrow \infty} \int_{B^{\prime} \times B^{\prime \prime}} \chi_{1} \ldots \chi_{k} u_{j}^{1} \Delta u_{j}^{2} \wedge \cdots \wedge \Delta u_{j}^{l-1} \wedge \Delta u_{j}^{l} \wedge \Delta u^{l+1} \wedge \cdots \wedge \Delta u^{k} \wedge T \wedge \alpha,
\end{aligned}
$$

which gives (3.16). The other terms involve $d_{0} \chi_{t} \wedge d_{1} u^{t}+d_{0} u^{t} \wedge d_{1} \chi_{t}+u^{t} \triangle \chi_{t}$ for at least one index $t$. It suffices to show that these terms do not give any contribution in the inequalities.

Note that the support of all derivatives of $\chi_{t}$ is disjoint from the unbounded locus of $u^{t}$ when $u^{t}$ is unbounded, and contained in $W_{t} \backslash \bar{W}_{t}^{\prime}$ when $u^{t}$ is bounded. In the first case, the number $m$ of unbounded functions is therefore replaced by $m-1$. Whereas in the second case $u_{j}^{t}=u^{t}$ is constant and smooth on the support of the derivatives of $\chi_{t}$, so $k$ can be replaced by $k-1$. By induction on $k+m$ and the polarization identity (3.4), the limit of the terms involving derivatives of $\chi_{t}$ is equal on both sides to the corresponding terms obtained by suppressing all indices $j$.

Let $k=p=n$ and $T$ be a constant in Theorems 1.1 and 1.2. Then we can get the following conclusion for the positive measure $\Delta u^{1} \wedge \cdots \wedge \Delta u^{n}$.

Corollary 3.2 Let $u^{1}, \ldots, u^{n}$ be PSH functions in $\Omega$ such that

$$
\mathcal{H}_{4 n-4 m+1}\left(L\left(u^{t_{1}}\right) \cap \ldots \cap L\left(u^{t_{m}}\right)\right)=0
$$

for all choices of indices $t_{1}<\ldots<t_{m}$ in $\{1, \ldots, n\}$. Then the quaternionic MongeAmpère measure $\Delta u^{1} \wedge \cdots \wedge \Delta u^{n}$ is well defined and has locally finite mass in $\Omega$. Moreover, let $\left\{u_{j}^{1}\right\}_{j \in \mathbb{N}}, \ldots,\left\{u_{j}^{n}\right\}_{j \in \mathbb{N}}$ be decreasing sequences of PSH functions in $\Omega$ such that $\lim _{j \rightarrow \infty} u_{j}^{i}=u^{i}$, pointwise in $\Omega, i=1, \ldots, n$. Then $\Delta u_{j}^{1} \wedge \cdots \wedge \Delta u_{j}^{n}$ converges weakly to $\Delta u^{1} \wedge \cdots \wedge \Delta u^{n}$ as $j \rightarrow \infty$.

\section{Generalized Lelong number}

For a closed positive $(2 n-2 p)$-current $T, a \in \Omega$, the Lelong number of $T$ at point $a$ was defined in [19] as the limit

$$
v_{a}(T):=\lim _{r \rightarrow 0+} \frac{\sigma_{T}(a, r)}{r^{4 p}},
$$

where

$$
\sigma_{T}(a, r):=\int_{B(a, r)} T \wedge \beta_{n}^{p}
$$


for small $r$. We showed that $\frac{\sigma_{T}(a, r)}{r^{4} p}$ is an increasing function of $r$.

Let $\varphi=-\|q-a\|^{-2}$, which is the fundamental solution of quaternionic Monge-Ampère operator, the generalized Lelong number $v(T, \varphi)$ given by (1.3) is just the Lelong number $v_{a}(T)$ of $T$ at point $a$. This is because:

$$
\begin{aligned}
v\left(T,-\|q-a\|^{-2}\right) & =\lim _{r \rightarrow-\infty} v\left(T,-\|q-a\|^{-2}, r\right)=\lim _{r \rightarrow 0} v\left(T,-\|q-a\|^{-2},-r^{-2}\right) \\
& =\lim _{r \rightarrow 0} \int_{\left\{-\|q-a\|^{-2}<-r^{-2}\right\}} T \wedge\left(\triangle\left(-\|q-a\|^{-2}\right)\right)^{p} \\
& =\lim _{r \rightarrow 0} \int_{B(a, r)} T \wedge\left(\triangle\left(-\|q-a\|^{-2}\right)\right)^{p}=v_{a}(T) .
\end{aligned}
$$

The Lelong-Jensen-type formula we established in [19, Theorem 5.1] also holds for the unbounded case, that is, the requirement of local boundedness of $V$ in Lelong-Jensen-type formula is not necessary. Recall that the quaternionic boundary measure associated with $\varphi$ is the nonnegative Borel measure $\mu_{\varphi, r}$ defined by:

$$
\mu_{\varphi, r}=\triangle_{n}\left(\varphi_{r}\right)-\chi_{\Omega \backslash B_{\varphi}(r)} \triangle_{n} \varphi,
$$

where $\varphi_{r}:=\max \{\varphi, r\}$ and $\triangle_{n} \varphi$ is the coefficient of the $2 n$-current $(\Delta \varphi)^{n}$. The measure $\mu_{\varphi, r}$ supports on $S_{\varphi}(r):=\{q \in \Omega ; \varphi(q)=r\}$.

Theorem 4.1 (Lelong-Jensen-type formula) Let $\Omega$ be a quaternionic strictly pseudoconvex domain. Let $\varphi: \Omega \rightarrow[-\infty,+\infty)$ be a continuous $P S H$ function, satisfying that, there exists a real number $R$ such that $B_{\varphi}(R) \Subset \Omega$. Let $V$ be a $P S H$ function in $\Omega$, then

$$
\begin{aligned}
\mu_{\varphi, r}(V)-\int_{B_{\varphi}(r)} V(\Delta \varphi)^{n} & =\int_{B_{\varphi}(r)}(r-\varphi) \Delta V \wedge(\Delta \varphi)^{n-1} \\
& =\int_{-\infty}^{r} \mathrm{~d} t \int_{B_{\varphi}(t)} \Delta V \wedge(\Delta \varphi)^{n-1} \\
& =\int_{-\infty}^{r} v(\Delta V, \varphi, t) \mathrm{d} t
\end{aligned}
$$

where $B_{\varphi}(r)$ is given by (1.5).

Proof For arbitrary $V \in P S H(\Omega)$, let $V_{h}$ be a decreasing sequence of smooth functions such that $V=\lim _{h \rightarrow 0} V_{h}$ with $V_{h} \in P S H\left(\Omega^{\prime}\right), \Omega^{\prime} \Subset \Omega$. Then $\triangle V_{h} \wedge(\triangle \varphi)^{n-1}$ converges weakly to $\triangle V \wedge(\triangle \varphi)^{n-1}$ by Corollary 3.2. So by Fatou lemma we have

$$
\liminf _{h \rightarrow 0} \int_{B_{\varphi}(r)}(r-\varphi) \Delta V_{h} \wedge(\Delta \varphi)^{n-1} \geq \int_{B_{\varphi}(r)}(r-\varphi) \Delta V \wedge(\triangle \varphi)^{n-1}
$$

with equality if $\varphi$ is finite. $\int_{B_{\varphi}(r)} V_{h}(\triangle \varphi)^{n} \rightarrow \int_{B_{\varphi}(r)} V(\triangle \varphi)^{n}$ and $\mu_{\varphi, r}\left(V_{h}\right) \rightarrow \mu_{\varphi, r}(V)$ by the monotone convergence theorem. Apply (4.3) to $V_{h}$ with $\varphi$ continuous and let $h \rightarrow 0$ to get

$$
\mu_{\varphi, r}(V)-\int_{B_{\varphi}(r)} V(\triangle \varphi)^{n} \geq \int_{B_{\varphi}(r)}(r-\varphi) \triangle V \wedge(\triangle \varphi)^{n-1},
$$

with equality if $\varphi$ is finite. On the other hand, for $V$ smooth and $l$ sufficiently large, in the proof of Theorem 5.1 in [19] we have

$$
\mu_{\varphi, r}(V)-\int_{B_{\varphi}(r)} V\left(\Delta \varphi_{-l}\right)^{n}=\int_{-l}^{r} \mathrm{~d} t \int_{B_{\varphi}(t)} \Delta V \wedge\left(\Delta \varphi_{-l}\right)^{n-1} .
$$


Now apply (4.4) to $V_{h}$ and let $h \rightarrow 0$ then we get (4.4) for arbitrary $V$. Letting $l \rightarrow+\infty$ on both side of (4.4), and noting that

$$
\int_{B_{\varphi}(r)}-V(\triangle \varphi)^{n} \leq \liminf _{l \rightarrow+\infty} \int_{B_{\varphi}(r)}-V\left(\Delta \varphi_{-l}\right)^{n}
$$

by Fatou lemma, we finally get the converse inequality

$$
\mu_{\varphi, r}(V)-\int_{B_{\varphi}(r)} V(\Delta \varphi)^{n} \leq \int_{-\infty}^{r} \mathrm{~d} t \int_{B_{\varphi}(t)} \Delta V \wedge(\Delta \varphi)^{n-1} .
$$

The following is some relationship between the generalized Lelong number and the quaternionic boundary measure.

Proposition 4.1 Suppose that $\Omega$ is a quaternionic strictly pseudoconvex domain, $\varphi$ is a continuous $P S H$ function satisfying that, there exists a real number $R$ such that $B_{\varphi}(R) \Subset \Omega$. Let $V \in P S H(\Omega)$. Assume that $(\triangle \varphi)^{n}=0$ on $\Omega \backslash S_{\varphi}(-\infty)$. Then $r \mapsto \mu_{\varphi, r}(V)$ is a convex increasing function of $r$ and the generalized Lelong number $v(\triangle V, \varphi)$ is given by:

$$
v(\triangle V, \varphi)=\lim _{r \rightarrow-\infty} \frac{\mu_{\varphi, r}(V)}{r} .
$$

Proof For $r<r_{0}$, by (4.3) and (1.4) we have

$$
\mu_{\varphi, r}(V)=\mu_{\varphi, r_{0}}(V)+\int_{r_{0}}^{r} v(\Delta V, \varphi, t) \mathrm{d} t .
$$

It follows from $v(\triangle V, \varphi, t)$ being increasing and nonnegative that $r \mapsto \mu_{\varphi, r}(V)$ is increasing and

$$
\begin{aligned}
\lim _{r \rightarrow-\infty} \frac{\mu_{\varphi, r}(V)}{r} & =\lim _{r \rightarrow-\infty} \frac{\mu_{\varphi, r_{0}}(V)+\int_{r_{0}}^{r} v(\Delta V, \varphi, t) \mathrm{d} t}{r} \\
& =\lim _{r \rightarrow-\infty} v(\Delta V, \varphi, r)=v(\Delta V, \varphi) .
\end{aligned}
$$

For $a \in \Omega$, the quaternionic pluri-Green function with pole at $a$ is defined as:

$$
g_{\Omega}(q, a)=\sup \left\{u(q): u \in \operatorname{PSH}(\Omega,[-\infty, 0)) ; u(q)+\frac{1}{\|q-a\|^{2}} \leq O(1) \text { as } q \rightarrow a\right\} .
$$

The quaternionic pluri-Green function $g_{\Omega}(q, a)$ is maximal and continuous in $\Omega \backslash\{a\}$. This can be proved by using the method as in the case of real $k$-Green function (cf. Appendix A. in [17]).

In Lelong-Jensen-type formula (4.3), let $\varphi$ be $g=g_{\Omega}(\cdot, a)$, we have

$$
V(a)=\frac{1}{C_{n}} \mu_{g, r}(V)-\frac{1}{C_{n}} \int_{B_{g}(r)}(r-g) \Delta V \wedge(\triangle g)^{n-1},
$$

where $C_{n}$ is a positive constant such that $(\triangle g)^{n}=C_{n} \delta_{a}$. In particular, if $V$ is pluriharmonic in $B_{g}(r)$, then $\triangle V=0$. So $V(a)=\frac{1}{C_{n}} \mu_{g, r}(V)$. In the special case $n=1$,

$$
V(a)=\frac{1}{C_{n}} \int_{S_{g}(r)} V \sum_{i j} \delta_{01}^{i j} \nabla_{j 1} g \cdot n_{i 0} \mathrm{~d} S,
$$


where $S_{g}(r)=\{q \in \Omega: g(q)=r\}$ and $n_{i 0}$ is given by (2.8). This is the Poisson integral formula and $\sum_{i j} \delta_{01}^{i j} \nabla_{j 1} g n_{i 0} \mathrm{~d} S$ is the harmonic measure. Therefore, the Lelong-Jensentype formula can be regarded as a higher-dimensional version of Poisson integral formula.

We can define quaternionic limit boundary measure associated to $\varphi$ and prove the comparison theorem for the quaternionic limit boundary measure as in the case of real $k$-Hessian operator (see Section 4 in [17] for detailed discussion).

By repeating the methods for complex PSH functions and positive currents (cf. $[8,11]$ ), one can get the following two Demailly's comparison theorems for the generalized Lelong number.

Corollary 4.1 (First comparison theorem) Let $\Omega$ be a quaternionic strictly pseudoconvex domain and let $T$ be a closed positive $(p, p)$-current. Let $\varphi, \psi$ be continuous PSH functions such that each one satisfies the hypotheses of Theorem 1.1 and that

$$
l=\lim \sup \frac{\psi(q)}{\varphi(q)}<+\infty \text { as } q \in \operatorname{Supp} T \text { and } \varphi(q) \rightarrow-\infty .
$$

Then $v(T, \psi) \leq l^{p} v(T, \varphi)$, and the equality holds if $l=\lim \frac{\psi}{\varphi}$.

Corollary 4.2 (Second comparison theorem) Let $\Omega$ be a quaternionic strictly pseudoconvex domain and let $u_{1}, \ldots, u_{k}$ and $v_{1}, \ldots, v_{k}$ be PSH functions such that each $k$-tuple satisfies the hypotheses of Theorem 1.1 with respect to $T$, where $T$ is a closed positive $(p, p)$-current in $\Omega$. Suppose that $u_{j}=-\infty$ on Supp $T \cap \varphi^{-1}(-\infty)$ and that

$$
l_{j}:=\lim \sup \frac{v_{j}(q)}{u_{j}(q)}<+\infty, \text { where } z \in \operatorname{Supp} T \backslash u_{j}^{-1}(-\infty), \varphi(q) \rightarrow-\infty \text {. }
$$

Then

$$
v\left(\Delta v_{1} \wedge \ldots \wedge \Delta v_{k} \wedge T, \varphi\right) \leq l_{1} \ldots l_{k} v\left(\Delta u_{1} \wedge \ldots \wedge \Delta u_{k} \wedge T, \varphi\right)
$$

Acknowledgements This work is supported by National Nature Science Foundation in China (Nos. 11401390; 11571305).

\section{References}

1. Alesker, S.: Non-commutative linear algebra and plurisubharmonic functions of quaternionic variables. Bull. Sci. Math. 127(1), 1-35 (2003)

2. Alesker, S.: Valuations on convex sets, non-commutative determinants, and pluripotential theory. Adv. Math. 195(2), 561-595 (2005)

3. Alesker, S.: Pluripotential theory on quaternionic manifolds. J. Geom. Phys. 62(5), 1189-1206 (2012)

4. Alesker, S.: Solvability of the quaternionic Monge-Ampère equation on compact manifolds with a flat hyperKähler metric. Adv. Math. 241, 192-219 (2013)

5. Alesker, S., Shelukhin, E.: On a uniform estimate for the quaternionic Calabi problem. Israel J. Math. 197(1), 309-327 (2013)

6. Alesker, S., Verbitsky, M.: Quaternionic Monge-Ampère equation and Calabi problem for HKTmanifolds. Israel J. Math. 176, 109-138 (2010)

7. Bedford, E., Taylor, B.A.: A new capacity for plurisubharmonic functions. Acta Math. 149(1-2), 1-40 (1982)

8. Demailly, J.-P.: Complex Analytic and Differential Geometry. Book available online at: http://wwwfourier.ujf-grenoble.fr/ demailly/documents.html

9. Demailly, J.-P.: Mesures de Monge-Ampère et mesures pluriharmoniques. Math. Z. 194(4), 519-564 (1987)

10. Demailly, J.-P.: Potential Theory in Several Complex Variables. (1989). http://www-fourier.ujf-grenoble. $\mathrm{fr} /$ demailly/documents.html 
11. Demailly, J.P.: Monge-Ampère operators, Lelong numbers and intersection theory. In: Ancona, V., Silva, A. (eds.) Complex Analysis and Geometry. University Series in Mathematics, pp. 115-193. Plenum, New York (1993)

12. Harvey, R.F., Lawson Jr., H.B.: Duality of positive currents and plurisubharmonic functions in calibrated geometry. Am. J. Math. 131(5), 1211-1239 (2009)

13. Kang, Q., Wang, W.: On Penrose integral formula and series expansion of $k$-regular functions on the quaternionic space $\mathbb{H}^{n}$. J. Geom. Phys. 64, 192-208 (2013)

14. Verbitsky, M.: Balanced HKT metrics and strong HKT metrics on hypercomplex manifolds. Math. Res. Lett. 16(4), 735-752 (2009)

15. Wan, D.: The continuity and range of the quaternionic Monge-Ampère operator on quaternionic space. Math. Z. 285, 461-478 (2017)

16. Wan, D., Kang, Q.: Potential theory for quaternionic plurisubharmonic functions. Michigan Math. J. 66, 3-20 (2017)

17. Wan, D., Wang, W.: Lelong-Jensen type formula, $k$-Hessian boundary measure and Lelong number for $k$-convex functions. J. Math. Pures Appl. 99(6), 635-654 (2013)

18. Wan, D., Wang, W.: Viscosity solutions to quaternionic Monge-Ampère equations. Nonlinear Anal. 140, 69-81 (2016)

19. Wan, D., Wang, W.: On the quaternionic Monge-Ampère operator, closed positive currents and LelongJensen type formula on the quaternionic space. Bull. Sci. Math. 141(4), 267-311 (2017)

20. Wan, D., Zhang, W.: Quasicontinuity and maximality of quaternionic plurisubharmonic functions. J. Math. Anal. Appl. 424, 86-103 (2015)

21. Wang, W.: On non-homogeneous Cauchy-Fueter equations and Hartogs' phenomenon in several quaternionic variables. J. Geom. Phys. 58(9), 1203-1210 (2008)

22. Wang, W.: The $k$-Cauchy-Fueter complex, Penrose transformation and Hartogs phenomenon for quaternionic $k$-regular functions. J. Geom. Phys. 60(3), 513-530 (2010)

23. Wang, W.: On the optimal control method in quaternionic analysis. Bull. Sci. Math. 135(8), 988-1010 (2011)

24. Wang, W.: The tangential Cauchy-Fueter complex on the quaternionic Heisenberg group. J. Geom. Phys. 61(1), 363-380 (2011)

25. Wang, W.: On quaternionic complexes over unimodular quaternionic manifolds. Differ. Geom. Appl. 58, $227-253$ (2018) 